\title{
Intrauterine insemination or in vitro fertilization for the treatment of Poseidon group 3 and unexplained subfertility: A propensity score matching study
}

\section{Yixuan Wu}

The Third Affiliated Hospital Of Guangzhou Medical University

Haiying Liu ( $\sim$ liuhaiying0606@163.com )

The Third Affiliated Hospital of Guangzhou Medical University

Jianqiao Liu

The Third Affiliated Hospital Of Guangzhou Medical University

\section{Research}

Keywords: unexplained infertility, poor ovarian reserve, treatment, in vitro fertilization, intrauterine insemination

Posted Date: August 4th, 2021

DOI: https://doi.org/10.21203/rs.3.rs-741836/v1

License: (c) (1) This work is licensed under a Creative Commons Attribution 4.0 International License. Read Full License 


\section{Abstract \\ Background}

Although it is becoming an increasingly significant indication for in-vitro fertilization (IVF), no studies have been done to examine the efficacy of IVF and intrauterine insemination (IUI) for the treatment of young patients with unexplained infertility and low ovarian reserve. The goal of this research was to compare the efficacy of IVF with IUI on Poseidon group 3 patients with unexplained infertility (PG3\&UI).

\section{Methods}

This was a retrospective analysis of PG3\&UI patients who had IVF/intracytoplasmic sperm injection (ICSI) or IUI at the Third Affiliated Hospital of Guangzhou Medical University between January 1, 2015, and March 31, 2021. To equalize the baseline characteristics of the IVF and IUI groups, propensity score matching (PSM) was utilized. Intention-to-treat (ITT) and per-protocol (PP) analyses were used to compare the differences in live births. To discover variations in time to biochemical pregnancy leading to live birth, Kaplan-Meier curves were produced. To evaluate the expenses per live birth between two procedures, a cost-effective analysis was done.

\section{Results}

According to ITT analysis, the live birth rate for the IVF group was substantially higher than the cumulative live birth rate (CLBR) for the IUI group (22.6\% (38/168) vs. $11.3 \%(19 / 168), \mathrm{RR} 2.00,95 \% \mathrm{Cl}$ $1.20-3.32, P=0.006)$. In the PP analysis, the live birth rate was $23.0 \%(38 / 165)$ in the IVF group and $11.7 \%(19 / 162)$ in the IUI group ( $R R=1.96,95 \% \mathrm{Cl} 1.18-3.26, P=0.007)$. When censored at 365 days, the Kaplan-Meier analysis revealed that the IVF group had a higher live birth rate than the IUI group (log-rank test $\left.\chi^{2}=6.025 ; P=0.014\right)$. However, when the two groups were censored at 180 days, the live birth rates were not substantially different (log-rank test $\chi^{2}=3.847 ; P=0.05$ ). The number of visits per live birth in the

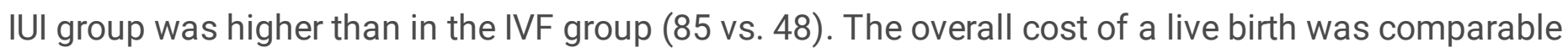
across the two groups ( $¥ 132242$ vs. $¥ 131611$ ), while the medical expenses for a live delivery from IVF were higher than those from IUI (( $¥ 118955$ vs. $¥ 108279)$.

\section{Conclusions}

The livebirth rate per IVF cycle with at most one embryo transfer is higher than the CLBR of IUI, with fewer hospital visits and similar expenses.

\section{Background}


Failure to conceive despite a year of regular unprotected intercourse in couples without anovulation, semen abnormalities, tubal disease, or other identifiable infertility reasons is classified as unexplained infertility. It accounts for around $30-40 \%$ of causes of infertility[1]. IUI and IVF are the most frequent assisted reproductive technologies(ART) used to treat unexplained infertility. Farquhar et al demonstrated that women who underwent three cycles of IUI with ovarian stimulation (IUI/OS) had a higher CLBR than women who underwent three cycles of expectant management. Women with unexplained infertility should undergo 3 to 4 cycles of IUI with clomiphene or letrozole [1].

Many prior types of research evaluated the efficacy of IVF with IUI or expectant management for unexplained infertility. In a randomized controlled trial (RCT) involving 258 couples, Goverde et al. compared the pregnancy rates of IVF and IUI. They concluded that the IVF group had a greater pregnancy rate each cycle than the IUI group (either IUI alone or IUI/OS). The cumulative pregnancy rate for 6 cycles of IVF, on the other hand, was equivalent to that of IUI[2]. Custers et al compared the effectiveness of a single cycle of IVF with a single embryo transfer against three cycles of IUI/OS. They demonstrated that the two groups had comparable rates of ongoing pregnancy and multiple pregnancies[3]. Elzeiny evaluated the effects of IVF and IUI in couples with unexplained infertility when only 2-3 mature follicles were stimulated by the same dosage of gonadotropin. Their study demonstrated that the IVF group had a greater live birth rate and lower cost per live birth[4]. Goldman etc. conducted a RCT in women $\geq 38$ years with unexplained infertility to evaluate therapy begun with IUI/OS vs. immediate IVF. They discovered that after two cycles of treatment, IVF was associated with a greater CLBR[5].

The previous studies' findings are unclear, thus whether IVF is preferable to IUI for the treatment of unexplained infertility remains debatable. Furthermore, these trials were done at a time when IVF live birth rates were substantially lower than they are now. Due to various advancements in ovarian stimulation and embryo culture technologies during the last two decades, IVF live birth rates have improved considerably[6, 7]. In comparison, IUI livebirth rates have been constant throughout the last few decades. Furthermore, no studies have been done to examine the efficacy of IVF and IUI on young patients with unexplained infertility who have an inadequate ovarian reserve, although this is becoming an increasingly significant indication for IVF[7].

As a result, the current study sought to compare the efficacy of IVF with IUI in Poseidon group 3 patients with unexplained infertility (PG3\&UI).

\section{Materials And Methods}

\section{Methods}

This was a retrospective analysis of PG3\&UI patients who had IVF/ ICSI or IUI at Third Affiliated Hospital of Guangzhou Medical University between January 1, 2015, and March 31, 2021. The study's goal was to assess the efficacy of IVF and IUI for the treatment of PG3\&UI patients. The Ethics Committee at the Third Affiliated Hospital of Guangzhou Medical University authorized the study. 


\section{Study Population}

All female Poseidon group 3 patients who had IVF or IUI in the Department of Reproductive Medicine, the Third Affiliated Hospital of Guangzhou Medical University, were screened. The following were the inclusion criteria: (1) Poseidon group 3, i.e., female age $<35$ years and $\mathrm{AMH}<1.2 \mathrm{ng} / \mathrm{ml}$; (2) Unexplained infertility,

which was defined as a menstrual cycle of 21-35 days; bilateral tubes patent confirmed by the hysterosalpingogram or laparoscopy; normal sperm analysis (concentration $\geq 15$ million per $\mathrm{mL}$ and progressive motility $\geq 32 \%$ ) and normal sexual function. (3) Only the first oocyte retrieval (OR) cycle with the first embryo transfer (ET) cycle (if there were embryos available for transfer) was included in the research for IVF patients. For IUI groups, all eligible patients' cycles were included in the analysis.

The exclusion criteria included (1) recurrent miscarriage; (2) ovarian tumors/cysts;(3) atypical endometrial hyperplasia;(4) cervical intraepithelial neoplasm (CIN) I-III; (5) chromosomal abnormalities;(6) intrauterine adhesion and (7) cycles with donor sperm.

\section{Treatment protocol}

For ovarian stimulation in IVF cycles, three protocols were used: mild stimulation, agonist, and antagonist. On cycle days 2-3, patients began mild ovarian stimulation with FSH $150 \mathrm{lU} /$ day and clomiphene citrate (CC, 100-150 mg/day). When a 14-mm diameter leading follicle appeared, an antagonist was employed to inhibit the luteinized hormone $(\mathrm{LH})$ surge. The antagonist protocol was similar to the mild stimulation protocol, except that the ovarian stimulation dosage was 150-300 IU of FSH without clomiphene citrate at the start. For the agonist protocol, triptorelin acetate $1.0 \mathrm{mg}$ was given in the mid-luteal phase of the previous cycle, followed by $150-300$ IU of FSH commencing 14 days following downregulation. When there were three 17-mm lead follicles, 250 ugs of recombinant hCG was administered subcutaneously. The oocytes were retrieved $36 \mathrm{~h}$ later, and the embryos were transferred 3-5 days later. All embryos were frozen for the $\mathrm{CC}$ cycles, and embryo transfer was done in another cycle, either natural or artificial. For luteal-phase support, 90 mg vaginal progesterone (Crinone, Merck Serono, England) was administered once daily.

Natural or stimulation protocols were employed for IUI. CC (50 mg/day), letrozole ( $5 \mathrm{mg} /$ day), or gonadotropin (37.5-75 IU/day) was started on cycle days 2-4 for cycles for ovarian stimulation. On cycle days 10-12, ultrasound and blood hormone assays (including estradiol, LH, and progesterone) were conducted, and further monitoring was scheduled based on follicle size. When the lead follicle reached 18 mm, 10000 IU hCG was given, followed by IUI 36-h later. IUI was conducted $24 \mathrm{~h}$ after the LH rise in the cycle. Dydrogesterone (10 mg twice per day) was used to support the luteal phase during the ovarian stimulation cycle.

The serum $\beta$-hCG test was done 14 days following the ET or IUI, and luteal-phase support was continued until the tenth week in the case of an intrauterine pregnancy. 


\section{Outcomes}

The primary outcome was the number of live births per couple. The IVF group contained only live births from one oocyte retrieval with at most one ET, whereas the IUI group included live births from all eligible patients' cycles. Clinical pregnancy, early miscarriage, and ectopic pregnancy, time to biochemical pregnancy leading to live delivery, cost, and time spent per live birth were secondary outcomes.

\section{Definitions}

An intrauterine/extrauterine gestational sac identified by ultrasonography with positive serum $\beta$-hCG was considered as clinical pregnancy. Early miscarriage was defined as fetal growth stop or the absence of heart activity in the gestational sac within the first 12 weeks of pregnancy. The term "ongoing pregnancy" denoted a pregnancy that was more than 12-week-old and had heart activity. Live birth is defined as a pregnancy that continues with a live fetus after 28 weeks of gestation. Time to biochemical pregnancy leading to live birth defined as the time from enrollment (when the patients were registered and signed the consent to performed IVF/IUI) to the time of positive serum hCG test (usually tested 14 days after ET or $\mid U I)$.

\section{Cost-effectiveness analysis}

Medical and non-medical expenses are included in the expenditures. The medical costs for IVF included the enrollment examination, medications for ovarian stimulation, follicle monitoring, oocyte retrieval, sperm wash, embryo culture and transfer, embryo freezing and thawing in frozen-thawed ET cycles, and medication for luteal phase support until the pregnancy test. The cost of IUI includes the enrollment exam, medications for ovarian stimulation, follicle monitoring, sperm wash, IUI, and medication for luteal phase support till the pregnancy test. Non-medical expenditures included transportation, lodging, and time away from work. The total expenses per live birth were used to calculate cost-effectiveness.

\section{Statistical analysis}

Since this was retrospective research, the baseline characteristics of the IVF and IUI groups differed. As a result, PSM was employed to screen a group of patients so that the baseline characteristics of the two groups were identical. The propensity score was calculated using the multiple logistic regression models, with IVF vs. IUI as the dependent variable and female age, duration of infertility, AMH level, and BMI as independent factors. The PSM was carried out using a caliper width of 0.2 of the standard deviation (SD) of the logit of the propensity score and 1:1 matching by closest neighbor matching. SD for baseline variables before and after PSM was computed; an absolute value less than 0.1 indicated a minor imbalance.

To compare live birth rates, we ran the following analyses: ITT analysis of all patients in the two groups following PSM (one oocyte retrieval cycle with at most one ET vs. all IUI cycles); PP analysis excluding women who cancelled the oocyte retrieval or IUl; post-hoc sensitivity analysis comparing the effectiveness of two IUI cycles vs. one oocyte retrieval cycle with at most one ET, excluding 81 IUI patients performing 
$\leq 1$ cycle without live birth. Only the first two cycles of IUI were analyzed for individuals who had $\geq 3$ cycles of IUI. We also performed a post-hoc sensitivity analysis to evaluate the efficacy of 2 IUI cycles vs. one ET cycle, with only patients who had ET included in the IVF group.

SPSS version 22.0 software was used for statistical analysis (IBM, Armonk, NY, USA). The means of quantitative variables with homogeneous variance were compared using the Student's t-test. Quantitative variables with heterogeneous variance were represented as the median (1st and 3rd quartiles), and the Mann-Whitney $U$ test was used to compare the medians. Risk ratios (RR) with $95 \%$ confidence intervals (Cl) were computed for dichotomous variables, and the $\chi^{2}$ test was used to determine significance. A logrank (mantel-Cox) test was used to evaluate the time to biochemical pregnancy leading to live birth between the IUI and IVF groups using Kaplan-Meier curves. $P<0.05$ was deemed statistically significant.

\section{Results}

\section{Baseline characteristics of patients before and after PSM}

After screening, 278 IVF and 189 IUI participants were enrolled in the research. However, after PSM, there were only 168 patients in each group. Before PSM, the duration of infertility and AMH were substantially different $(|S D|>0.1)$ between the two groups. Female age, infertility duration, $A M H$, and BMI were all balanced between the two groups after matching. In the IVF and IUI groups, the female age was $31.0 \pm 2.5$ years and $30.9 \pm 2.4$ years, respectively $(|S D|<0.1)$. The IVF group had an AMH level of $0.82 \pm 0.28 \mathrm{ng} / \mathrm{mL}$, which was similar to the IUI group $(0.83 \pm 0.27 \mathrm{ng} / \mathrm{mL})(|S D|<0.1)$.

\section{Outcomes of patients after IVF and IUI}

Three of the 168 patients who began IVF had their oocyte retrieval terminated owing to poor ovarian response, 7 (4.2\%) had no oocytes retrieved, and 34 (20.2\%) had no transferable embryos. There were 63 fresh embryo transfers and 51 frozen embryo transfers. The frozen embryos from the first cycle had not been transferred to the remaining 10 patients since they had begun another IVF cycle

\section{Table 1 Characteristics of patients before and after PSM}




\section{After matching}

\begin{tabular}{|c|c|c|c|c|c|c|}
\hline & $\begin{array}{l}\text { IVF } \\
\text { group }\end{array}$ & $\begin{array}{l}\text { IUI } \\
\text { group }\end{array}$ & $\begin{array}{l}\text { Standardized } \\
\text { difference }\end{array}$ & $\begin{array}{l}\text { IVF } \\
\text { group }\end{array}$ & $\begin{array}{l}\text { IUI } \\
\text { group }\end{array}$ & $\begin{array}{l}\text { Standardized } \\
\text { difference }\end{array}$ \\
\hline$N$ & 278 & 189 & & 168 & 168 & \\
\hline $\begin{array}{l}\text { Female age } \\
\text { (year) }\end{array}$ & $31.0 \pm 2.6$ & $30.7 \pm 2.4$ & 0.095 & $31.0 \pm 2.5$ & $30.9 \pm 2.4$ & -0.005 \\
\hline $\begin{array}{l}\text { infertility } \\
\text { duration (year) }\end{array}$ & $3.94 \pm 2.2$ & $3.52 \pm 2.13$ & 0.190 & $3.72 \pm 1.93$ & $3.64 \pm 2.20$ & 0.034 \\
\hline $\mathrm{AMH}(\mathrm{ng} / \mathrm{mL})$ & $0.60 \pm 0.33$ & $0.86 \pm 0.27$ & -0.533 & $0.82 \pm 0.28$ & $0.83 \pm 0.27$ & -0.013 \\
\hline BMI $\left(\mathrm{kg} / \mathrm{m}^{2}\right)$ & $21.3 \pm 3.3$ & $21.5 \pm 3.1$ & -0.067 & $21.4 \pm 3.1$ & $21.3 \pm 2.8$ & 0.024 \\
\hline $\begin{array}{l}\text { No. of } \\
\text { previous } \\
\text { deliveries }\end{array}$ & $0.10 \pm 0.35$ & $0.13 \pm 0.35$ & NA & $0.13 \pm 0.37$ & $0.13 \pm 0.36$ & NA \\
\hline $\begin{array}{l}\text { Male age } \\
\text { (year) }\end{array}$ & $32.8 \pm 3.5$ & $32.6 \pm 3.9$ & NA & $32.9 \pm 3.6$ & $32.8 \pm 3.8$ & NA \\
\hline AFC & $6.4 \pm 2.6$ & $7.9 \pm 2.3$ & NA & $7.0 \pm 2.6$ & $7.7 \pm 2.2$ & NA \\
\hline 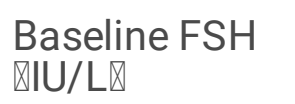 & $8.39 \pm 4.65$ & $6.84 \pm 2.63$ & NA & $7.65 \pm 4.10$ & $6.86 \pm 2.64$ & NA \\
\hline
\end{tabular}

Note『PSM for female age, infertility duration, $\mathrm{AMH}$ and BMI. NA=not applicable.

and had live births. The 114 embryo transfer cycles resulted in 38 live births (22.6\% per OR cycle and $33.3 \%$ each ET cycle), two ongoing pregnancies (beyond 12 weeks currently), six miscarriages, one ectopic pregnancy, and one induced abortion (due to major birth defect) (Fig 2 \& Table 2). Fourteen of the 168 patients who started IUI had their first IUI cycle terminated due to inadequate follicle growth, multiple follicle growth, or abnormal semen analysis, etc. (Fig 2). The remaining 154 IUI cycles resulted in 11 live births, 5 miscarriages, one onging and one ectopic pregnancy, and one fetal death. There were 87 patients starting cycle 2 , with 10 having IUls cancelled for various reasons. The 77 IUI cycles resulted in seven live births, one miscarriage, and one ectopic pregnancy. Only 23 patients

\section{Table 3 Livebirths by intention-to-treat and per-protocol analyses}




\begin{tabular}{|c|c|c|c|c|}
\hline & IVF & IUI & $\mathrm{RR}(95 \% \mathrm{Cl})$ & $P$ \\
\hline Intention-to-treat analysis & $22.6 \rrbracket 38 / 168 \rrbracket$ & $11.3 \rrbracket 19 / 168)$ & $2.00(1.20-3.32 \rrbracket$ & 0.006 \\
\hline Intention-to-treat analysis ${ }^{a}$ & $22.6 \rrbracket 38 / 168 \rrbracket$ & $6.7(19 / 284)$ & $3.38 \otimes 2.02-5.67)$ & 8.230E-7 \\
\hline Per-protocol analysis ${ }^{b}$ & $23.0 \rrbracket 38 / 165)$ & $11.7(19 / 162)$ & 1.96冈1.18-3.26ه & 0.007 \\
\hline Post-hoc per-protocol analysis ${ }^{c}$ & $23.0 \rrbracket 38 / 165)$ & $20.7(18 / 87)$ & $1.11 \otimes 0.68-1.83 \rrbracket$ & 0.675 \\
\hline Post-hoc per-protocol analysis ${ }^{d}$ & $33.3 \varangle 38 / 114)$ & $20.7(18 / 87)$ & $1.61 \llbracket 0.99-2.62)$ & 0.048 \\
\hline
\end{tabular}

Notes: a. One started cycle of IVF vs. one started cycle of IUI. b. Excluded 6 cycles in the IUI group (cancelled IUI) and 3 cycles in the IVF groups (cancelled the oocyte retrieval). c. Two IUI cycles vs. one oocyte retrieval cycle (Excluded $81 \mathrm{IUI}$ cycles that had $\leq 1$ cycle without live birth. For IUI patients with $\geq 3$ cycles of IUI, only the CLBR of the first two cycles were calculated. Exclude 3 IVF cycles that cancelled the oocyte retrieval). d. 2 IUI cycles vs. 1 embryo transfer cycle (Excluded $81 \mathrm{IUI}$ cycles that had $\leq 1$ cycle and no live birth. For IUI patients with $\geq 3$ cycles of IUI, only the cumulative live birth of the first two cycles were calculated. Only the 63 fresh ET and 51 frozen ET were included in the IVF groups).

higher live birth rate per cycle than the IUI group (22.6\% (38/168) vs. 6.7\% (19/284), RR 3.38, 95\% Cl 2.02$5.67, P=8.230 \mathrm{E}-7$; Table 2,3). In the PP analysis, which excluded 3 IVF patients who cancelled oocyte retrieval and $6 \mathrm{IUI}$ patients who canceled IUI, the livebirth rate in the IVF group was $23.0 \%(38 / 165)$ and $11.7 \%(19 / 162)$ in the IUI group $(\mathrm{RR}=1.96,95 \% \mathrm{Cl} 1.18-3.26, P=0.007)$. The effectiveness of one oocyte retrieval cycle and $2 \mathrm{IUI}$ cycles was assessed in the post-hoc sensitivity analysis. Patients with $\leq 1$ cycle of IUI without live birth were eliminated from the analysis in the IUI group. Only the CLBR of the first two cycles of IUI were computed for patients who had $\geq 3$ cycles of IUI. The live birth rate was not substantially different between the two groups in this situation (23.0\% (38/165) vs. $20.7 \%$ (18/87), RR $1.11,95 \% \mathrm{Cl} 0.68-1.83, P=0.675)$. When the efficacy of one ET cycle was compared to the efficacy of two IUI cycles, one ET cycle had a higher live birth rate than two IUI cycles (33.3\% (38/114) vs. 20.7\% (18/87), RR 1.61, 95\% Cl 1.61 0.99-2.62, $P=0.048$ ) (Tables $2 \& 3$ ).

\section{Time to biochemical pregnancy leading to live birth}

When censored at 365 days, the Kaplan-Meier analysis revealed that the IVF group had a higher live birth rate than the IUI group (log-rank test $\left.\chi^{2}=6.025 ; P=0.014\right)$. However, when the two groups were censored at 180 days, the live birth rates were not substantially different (log-rank test $\left.\chi^{2}=3.847 ; P=0.05\right)$. In all, 1612 visits were made in the IUI group while 1836 visits were made in the IVF group. The number of visits per live birth in the IUI group was higher than in the IVF group (85 vs. 48) (Table 4)

\section{Costs per live birth for IVF and IUI}

The overall cost of live birth was comparable across the two groups ( $¥ 132242$ vs. $¥ 131611$ ), while the medical expenses for a live delivery from IVF were higher than those from IUI (¥118955 vs. ¥108279). 
When compared to the IUI group, the expenditures per couple for IVF were substantially greater (¥31805 vs. ¥14885).

\section{Neonatal outcomes after IVF and IUI}

Six twins were born in the IVF group, while all live births in the IUI group were singletons. The infant outcomes were comparable between the two groups, including birth weight, height, gestational days, preterm delivery, and low birth weight. There was one birth defect in the IVF group and one still birth in the IUI group.

\section{Table 5 Cost-effectiveness analysis}

\begin{tabular}{clllll} 
Type of cycles & Total cost $(¥)$ & Livebirths & Cost/livebirth (\#) & couples & Cost/couple (¥) \\
\hline IUl total costs ${ }^{\text {a }}$ & 2500608 & 19 & 131611 & 168 & 14885 \\
\hline Medical costs & 2057308 & & 108279 & & 12246 \\
\hline Non-medical costs ${ }^{\text {b }}$ & 443300 & & 23332 & & 2639 \\
\hline IVF total costs & 5025209 & 38 & 132242 & $158^{c}$ & 31805 \\
\hline Medical costs & 4520309 & & 118955 & & 28610 \\
\hline Non-medical costs & 504900 & & 13287 & & 3196
\end{tabular}

Notes: a. total costs=medical costs + non-medical costs. b. Indirect costs=costs of transportation, lodging, and time away from work. c. Ten patients in the IVF groups weren't calculated in the cost. The frozen embryos from the first cycle had not been transferred since they had begun another IVF cycle and had live births.

Table 6 Neonatal outcomes after IVF and AlH 


\begin{tabular}{|c|c|c|c|}
\hline Newborn outcomes & IVF & IUI & $P$ \\
\hline & 38 & 19 & \\
\hline Singleton $\% \bowtie n \rrbracket$ & $84.6(32 / 38)$ & $100(19 / 19)$ & \multirow[t]{2}{*}{$0.164^{\circ}$} \\
\hline Twin $\% \rrbracket n \rrbracket$ & $15.4(6 / 38)$ & 0 & \\
\hline Birth weight $\llbracket g \rrbracket$ & $2956 \pm 676$ & $2886 \pm 811$ & 0.537 \\
\hline Birth height $\llbracket \mathrm{cm}$ ) & $47.5 \pm 8.1$ & $49.2 \pm 2.1$ & 0.356 \\
\hline Gestational days (day) & $268 \pm 19$ & $271 \pm 16$ & 0.490 \\
\hline Gestational weeks $<37 \%(\mathrm{n} \rrbracket$ & $15.8(6 / 38)$ & $26.3(5 / 19)$ & $0.478^{a}$ \\
\hline Gestational weeks $<32 \%(n \rrbracket$ & $7.9(3 / 38)$ & $5.3(1 / 19)$ & $1.000^{a}$ \\
\hline Birth weight $<2500 \mathrm{~g} \%(\mathrm{n} \rrbracket$ & $15.8(6 / 38)$ & $21.1(4 / 19)$ & $0.717^{a}$ \\
\hline Birth weight $<1500 \mathrm{~g} \%(\mathrm{n} \nabla$ & $2.6(1 / 38)$ & 0 & $1.000^{a}$ \\
\hline Fetal death $\%(\mathrm{n} \rrbracket$ & 0 & $5.3(1 / 19)$ & $0.333^{a}$ \\
\hline Birth defect \%(n区 & $5.3(1 / 38)$ & 0 & $1.000^{a}$ \\
\hline
\end{tabular}

a Fisher exact test was used.

\section{Discussions}

Expectant management, IUI/OS, and IVF are the three therapeutic options for unexplained infertility. Farquhar et al demonstrated that three cycles of IUI/OS outperformed three cycles of expectant care in CLBR for unexplained infertility [8] .However, the efficacy of IVF as compared to IUI is highly debated. When evaluating treatment options, five factors must be considered: The first is effectiveness (i.e., live birth rate or CLBR); the second is cost (including medical and non-medical costs); the third is time spending (time to live birth and time spent on treatment); the fourth is the patients' physical and psychological burden; and the last is maternal, fetal, and neonatal safety[9].

Several studies have been conducted to compare the efficacy of IVF with IUI. Angelique and her colleagues compared six cycles of IVF against six cycles of IUI and six cycles of IUI/OS. They observed that, whereas the IVF group had a higher live birth rate each cycle $(12.2 \%$ vs. $7.4 \%$ and $8.7 \%$, respectively; $P=0.09)$, the total live birth rates were comparable across the three groups. The dropout rate in the IVF group, on the other hand, was as high as $45 \%$ which would have an impact on the efficacy of IVF[2]. Three more studies $[3,10,11]$ compared the efficacy of one cycle of IVF to three cycles of IUI/OS and discovered no benefit to IVF. However, the first two studies[3, 11] failed to determine the sample size required to detect the difference in the primary outcome. The sample size in each group was only 58 and may be too small to make the difference significant. Although the third study calculated the sample size and planed 125 
couples for each arm, the study ended when only 207 couples were enrolled because of fund withdrawal. Therefore, these three studies were of low quality and the conclusions were not convincing enough[10].

The preceding trials were done at a time when the live birth rate of IVF was much lower (12-24.7\%) than it is now (42.23\% in China in 2018). The live birth rate of IUI has been pretty stable over the last two decades: $7-10 \%$ in the studies mentioned above vs. $10.7 \%$ in China in 2018 . As a result, with the current state of ART, it is critical to reassess the effectiveness of IVF vs. IUI/OS. According to the Chinese Society of Reproductive Medicine's (CSRM) 2018 Annual Report on Assisted Reproductive Technology (ART), poor ovarian reserve (POR) accounted for $11.84 \%$ of IVF indications[7]. POR patients who do not have additional infertility problems are a subset of individuals with unexplained infertility who have impaired ovarian function. Although POR has become an important indication for IVF, it is still uncertain if IVF is better than IUI for patients with unexplained infertility and POR (UIPOR). As a result, we conducted this study to compare the efficacy of IVF with IUI in patients with UIPOR.

We looked at patients in Poseidon group 3 (age $<35$ and $\mathrm{AMH}<1.2 \mathrm{ng} / \mathrm{mL}$ ) who had no other known reasons for infertility. To equalize the baseline features of the two groups, we employed the PSM. In the ITT analysis, our study found that the live birth rate in the IVF group was substantially higher than the CLBR in the IUI group (22.6 \% vs. $11.3 \%$, RR $2.00,95 \% \mathrm{Cl} 1.20-3.32, P=0.006)$. However, the mean cycles in the IUI groups were just 1.69. Because this is a retrospective, real-world study, comparing the efficacy of one IVF cycle with three IUI cycles is challenging, as it would be in a well-designed RCT. Because of their low ovarian reserve, POR patients are frequently concerned about their prognosis. They would prefer IVF as their first-line treatment since they feel it has a significantly greater pregnancy rate than IUI. As a result, UIPOR patients decline IUI after 1-2 unsuccessful cycles. IVF had a live birth rate of $22.6 \%$ per begun cycle, compared to $6.7 \%$ per initiated cycle in the IUI group. The CLBR of three cycles of IUI is thought to be equivalent to one cycle of IVF. In our facility, individuals with unexplained infertility are advised to undergo two cycles of IUI before doing IVF. So, we compared the CLBR of two IUI cycles in 87 patients to the LBR of one IVF cycle and discovered that the LBRs were identical in both groups: $20.7 \%$ vs. $23.0 \%$ ( $P$ $=0.675$ ). We should be very careful about this conclusion since individuals who had only one IUI cycle were omitted from the research, and their prognosis may have been poorer.

When evaluating a treatment approach, a cost-effectiveness analysis is critical. Goverde et al examined the CLBR for 6 cycles of IVF, 6 cycles of IUI, and 6 cycles of IUI/OS. They discovered that the IUI with natural cycle was the least expensive of the three procedures[2]. Tjon-Kon-Fat et al evaluate the expenses of three IVF-SET cycles to six IVF-MNC cycles vs. six IUI/OS cycles. When compared to IUI-COH (€5070), the mean expenses per couple for IVF-MNC (€8206) and IVF-SET (€7187) were substantially higher. When compared to IUI/OS, the cost of an extra live birth via IVF-SET would be €43 375[12]. Nandi et al. compared the efficacy of one IVF cycle to $3 \mathrm{IUI} / \mathrm{OS}$ cycles and determined that the expenses per livebirth for IVF were higher than for IUI, with a cost ratio of 1.3:1[10].

All three of the preceding studies solely analyzed medical expenses, demonstrating that IVF was more expensive than IUI. They did, however, overlook non-medical expenditures such as transportation, lodging, 
and time away from work. When we assessed the medical expenditures per live birth in our study, the costs for IVF were $¥ 10676$ greater than the costs for IUI. When the overall expenses per live birth, which included non-medical charges, were assessed, the prices for IVF and IUI were relatively similar. Our system didn't record the expenses beyond enrollment; therefore, we only computed the total expenditures from enrollment until the time of biochemical pregnancy.

Time to live birth and hospital visits on the therapy are two indicators used to assess the amount of time spent on fertility treatment. We didn't utilize the criterion "time to live birth" since it only included patients who had a live delivery and ignored the time spent by those who didn't. Because of the intricacy of IVF, the time to live delivery may be longer than with IUI. To compare the time spent for the two procedures, we utilized the Kaplan-Meier curves, which is recommended for evaluating the CLBR across time in clinical investigations[13]. The findings showed that when the two groups were censored at 180 days, the live birth rates were not substantially different. However, when censored at 365 days, IVF had a higher live birth rate than IUI (log-rank test $\left.\chi^{2}=6.025 ; P=0.014\right)$. There have been no prior studies that focused on hospital visits for fertility treatment. We tallied the hospital visits and discovered that IUI patients required more hospital visits per live delivery than IVF patients (85 vs. 48). The majority of patients have employment, and they frequently have to request time off for treatment, which has a detrimental impact on their productivity and income. Furthermore, repeated hospital visits exhaust and stress them. From this perspective, IUI is less patient-friendly than IVF.

The safety aspects included: complications of ovarian stimulation (e.g., ovarian hyperstimulation, OHSS), oocyte aspiration (e.g., pelvic hemorrhage), maternal and fetal problems during pregnancy (mostly multiple pregnancies and birth abnormalities), and natal complications (e.g., premature delivery). There were two occurrences of late OHSS in the IVF group and none in the IUI group in the current research. Except for 6 twins and one birth defect in the IVF group and 1 fetal death in the IUI group, the neonatal results were similar between the two groups.

The current study has the following advantages: first, it is the first to assess treatment options for Poseidon group 3 and unexplained infertility patients. Second, PSM was utilized to equalize the baseline features of the two groups, reducing the impact of baseline on live birth. Third, while assessing costeffectiveness, we included non-medical expenditures, which represent the couples' real costs. Finally, we compute the number of hospital visits per live birth, which represents the patients' physical and psychological stress.

The drawbacks are as follows: first, because this is retrospective research, it cannot adequately evaluate the efficacy of one IVF cycle with three cycles of IUI. In our study, the average number of IUI cycles was only 1.67. However, it is real-world research that represents the patients' and physicians' actual alternatives. Second, while assessing cost-effectiveness, the expenditures associated with pregnancy and delivery was not considered. Because there were six twin pregnancies in the IVF group, the expenses of IVF will be underestimated. 


\section{Conclusions}

In real-world research of Poseidon group 3 and unexplained infertility patients, the CLBR of IVF with at most one embryo transfer is greater than the CLBR of IUI, with fewer hospital visits, comparable expenditures, and time to biochemical pregnancy leading to live delivery when censoring at 180 days. A randomized controlled trial should be carried out to assess the efficacy of IVF and IUI for Poseidon group 3 and unexplained infertility patients.

\section{Abbreviations}

IVF: in vitro fertilization; IUI: intrauterine insemination; PG3\&UI :Poseidon group 3 patients with unexplained infertility; ICSI: intracytoplasmic sperm injection; PSM: propensity score matching; ITT: ntention-to-treat; PP: per-protocol; CLBR: cumulative live birth rate; ART: assisted reproductive technologies; IUI/OS :IUI with ovarian stimulation; RCT :randomized controlled trial; $\mathrm{AMH}$ : anti-müllerian hormone; OR: oocyte retrieval; ET: embryo transfer; CIN: cervical intraepithelial neoplasm; CC: clomiphene citrate; LH: luteinized hormone; hCG: human chorionic gonadotropin; SD: standard deviation; Cl: confidence intervals; AFC:antral follicle count; FSH: follicle-stimulating hormone.

\section{Declarations}

\section{Ethics approval and consent to participate}

This study was approved by the ethics committee of the Third Affiliated Hospital of Guangzhou Medical University. Each patient has signed informed consent on obtaining and analyzing their clinical data prior to the initiation of ART treatment.

\section{Consent for publication}

Not applicable.

\section{Availability of data and materials}

The data sets used and/or analyzed during the current study are available from the corresponding author on reasonable request.

\section{Competing interests}

The authors declare that they have no competing interests.

\section{Funding}

This study was supported by the the National Key Research and Development Program of China (No.2018YFC1003803囚to JQL) هScientific Research Program of The Third Affiliated Hospital of 
Guangzhou Medical University (No.2017Q15, to YXW) खNational Natural Science Foundation of China (No.81801532 to HYL) 『Guangzhou Science and Technology Plan Project $₫$ No.202102010076 to HYLه.

\section{Authors' contributions}

Conceptualization: Yixuan Wu, Jianqiao Liu; Methodology: Yixuan Wu; Formal analysis and investigation: Yixuan Wu; Writing - original draft preparation: Yixuan Wu; Review and editing: Haiying Liu; Funding acquisition: Jianqiao Liu; Resources: Haiying Liu; Supervision: Jianqiao Liu.

\section{Acknowledgments}

The study was performed under the auspices of the in vitro fertilization (IVF) unit of the Third Affiliated Hospital of Guangzhou Medical University. The authors would like to thank all the reviewers who participated in the review and MJEditor (www.mjeditor.com) for their linguistic assistance during the preparation of this manuscript.

\section{References}

1. Practice Committee of the American Society for Reproductive Medicine. Electronic address aao and Practice Committee of the American Society for Reproductive M. Evidence-based treatments for couples with unexplained infertility: a guideline. Fertil Steril. 2020;113:305-22.

2. Goverde AJ, McDonnell J, Vermeiden JP, Schats R, Rutten FF, Schoemaker J. Intrauterine insemination or in-vitro fertilisation in idiopathic subfertility and male subfertility: a randomised trial and cost-effectiveness analysis. Lancet. 2000;355:13-8.

3. Custers IM, Konig TE, Broekmans FJ, Hompes PG, Kaaijk E, Oosterhuis J, et al. Couples with unexplained subfertility and unfavorable prognosis: a randomized pilot trial comparing the effectiveness of in vitro fertilization with elective single embryo transfer versus intrauterine insemination with controlled ovarian stimulation. Fertil Steril. 2011;96:1107-11 e1.

4. Elzeiny H, Garrett C, Toledo M, Stern K, McBain J, Baker HW. A randomised controlled trial of intrauterine insemination versus in vitro fertilisation in patients with idiopathic or mild male infertility. Aust N Z J Obstet Gynaecol. 2014;54:156-61.

5. Goldman MB, Thornton KL, Ryley D, Alper MM, Fung JL, Hornstein MD, et al. A randomized clinical trial to determine optimal infertility treatment in older couples: the Forty and Over Treatment Trial (FORT-T). Fertil Steril. 2014;101:1574-81 e1-2.

6. Toner JP, Coddington CC, Doody K, Van Voorhis B, Seifer DB, Ball GD, et al. Society for Assisted Reproductive Technology and assisted reproductive technology in the United States: a 2016 update. Fertil Steril. 2016;106:541-6.

7. Jingwei Yang C, Deng X, Huang P, Liu C, Zhou Y, Feng, et al. Annual report On assisted reproductive technology of Chinese Society of Reproductive Medicine in 2018. Journal of Reproductive Medicine. 2021; 30: $419-25$. 
8. Farquhar CM, Liu E, Armstrong S, Arroll N, Lensen S, Brown J. Intrauterine insemination with ovarian stimulation versus expectant management for unexplained infertility (TUI): a pragmatic, open-label, randomised, controlled, two-centre trial. Lancet. 2018;391:441-50.

9. Mol BW, Tjon-Kon-Fat R, Kamphuis E, van Wely M. Unexplained infertility: Is it over-diagnosed and over-treated? Best Pract Res Clin Obstet Gynaecol. 2018;53:20-9.

10. Nandi A, Bhide P, Hooper R, Gudi A, Shah A, Khan K, et al. Intrauterine insemination with gonadotropin stimulation or in vitro fertilization for the treatment of unexplained subfertility: a randomized controlled trial. Fertil Steril. 2017; 107: 1329-35 e2.

11. van Rumste MM, Custers IM, van Wely M, Koks CA, van Weering HG, Beckers NG, et al. IVF with planned single-embryo transfer versus IUI with ovarian stimulation in couples with unexplained subfertility: an economic analysis. Reprod Biomed Online. 2014;28:336-42.

12. Tjon-Kon-Fat RI, Bensdorp AJ, Bossuyt PM, Koks C, Oosterhuis GJ, Hoek A, et al. Is IVF-served two different ways-more cost-effective than IUI with controlled ovarian hyperstimulation? Hum Reprod. 2015;30:2331-9.

13. Sunkara SK, Zheng W, D'Hooghe T, Longobardi S, Boivin J. Time as an outcome measure in fertilityrelated clinical studies: long-awaited. Hum Reprod. 2020;35:1732-9.

\section{Supplementary}

Due to technical limitations, Table 2 and 4 are only available as a download in the Supplemental Files section.

\section{Figures}




\section{IVF}

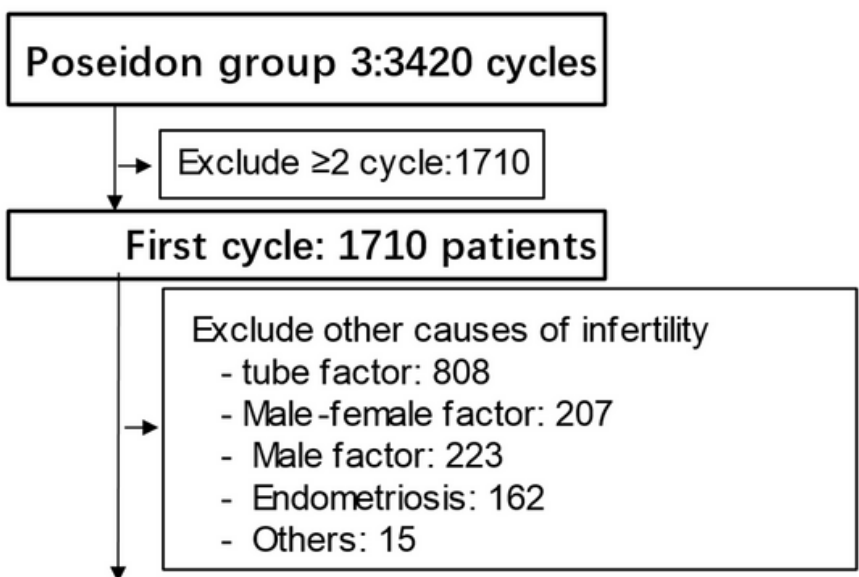

\section{Unexplained infertility: 295 patients}

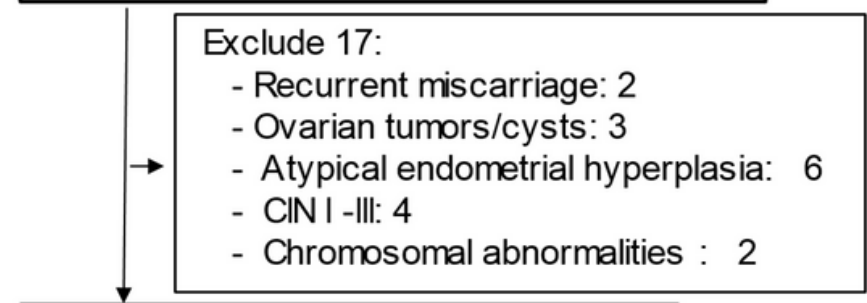

Included in study: 278 patients

\section{IUI}

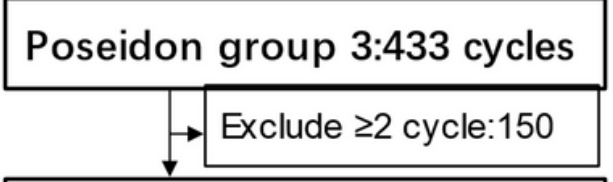

First cycle: 283 patients

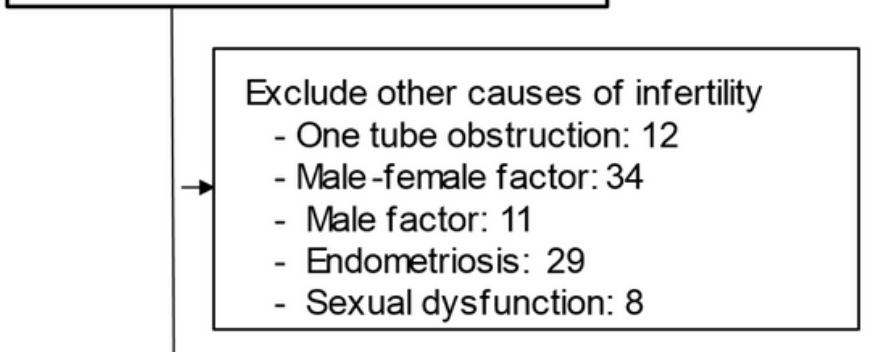

Unexplained infertility: 189 patients

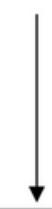

Included in study: 189 patients

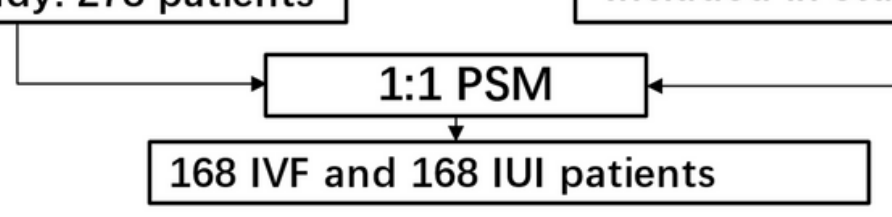

Figure 1

Process of screening patients 


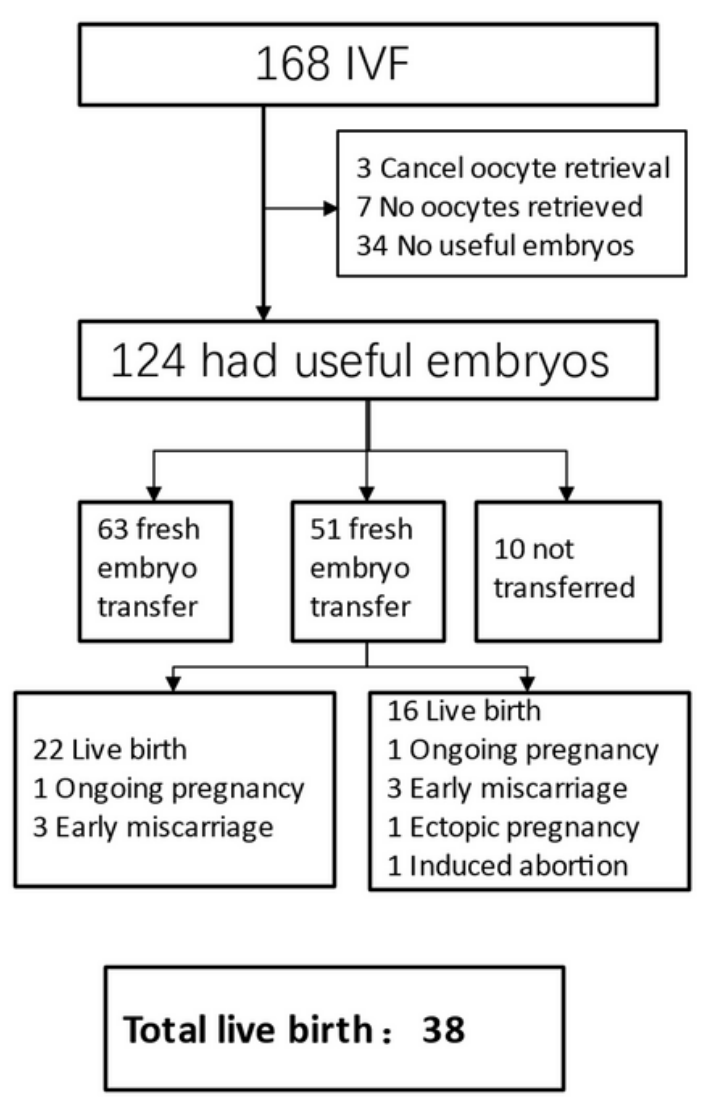

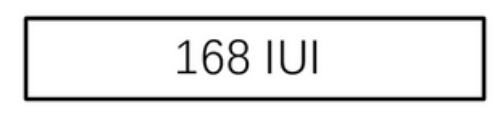

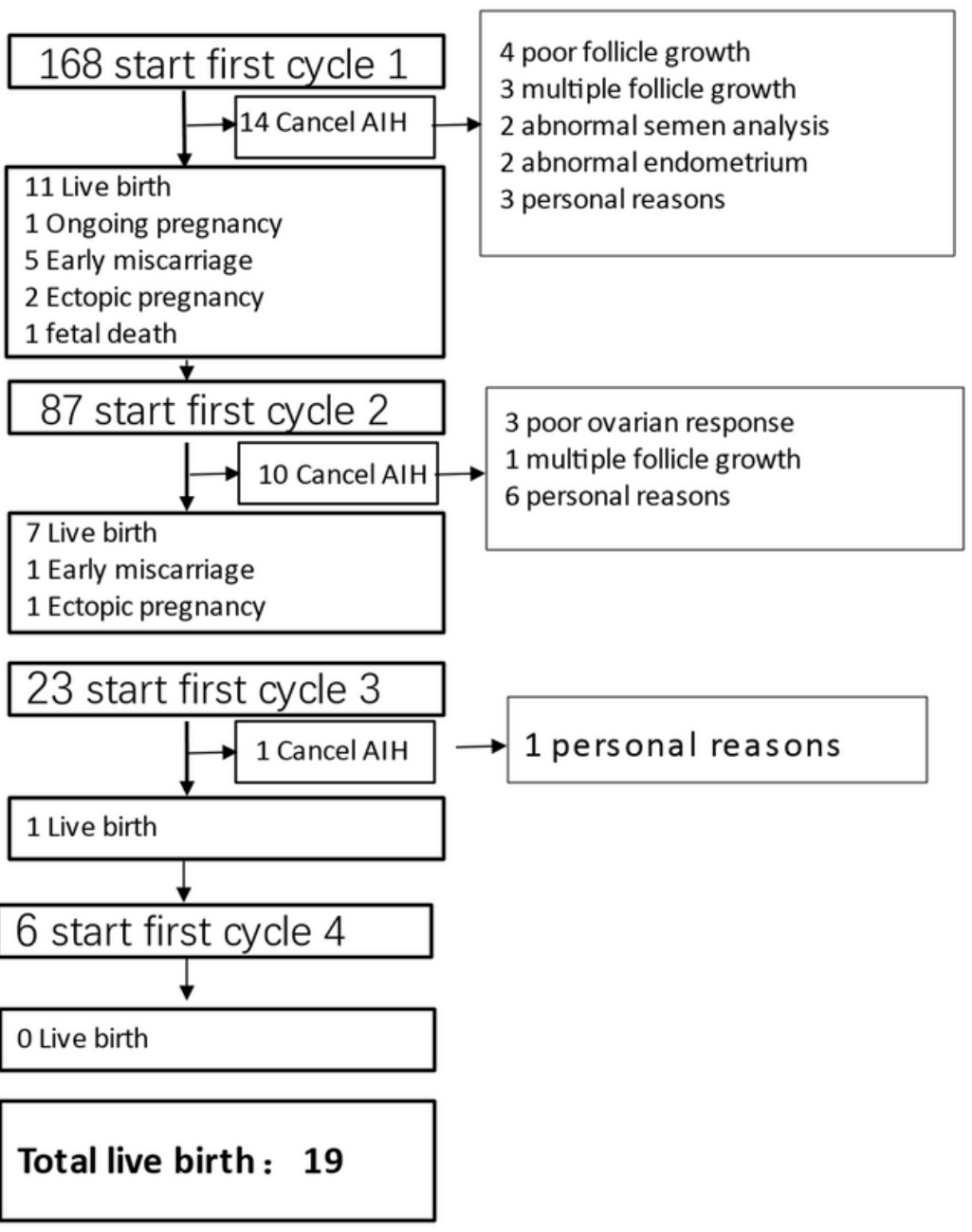

\section{Figure 2}




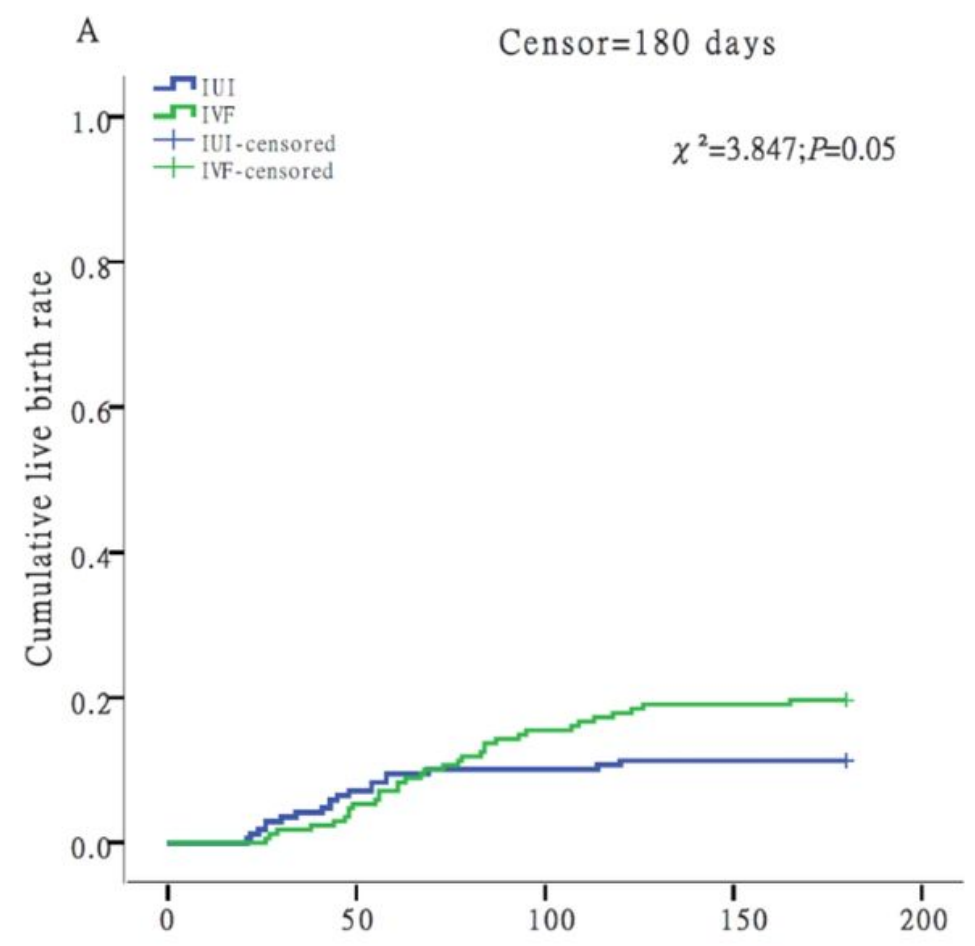

Time from enrollment to biochemical pregnancy leading to live birth (days)

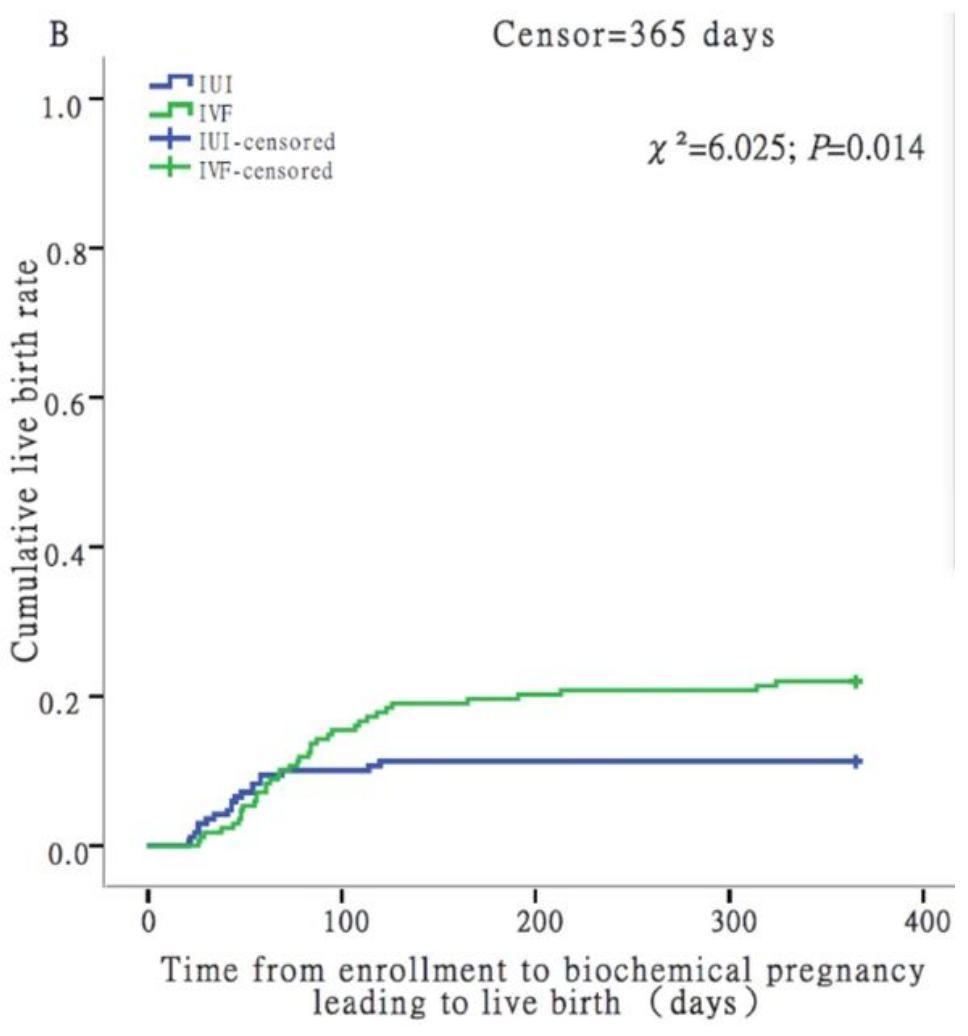

Figure 3

Time from enrollment to biochemical pregnancy leading to live birth in the IUI and IVF/ICSI groups For women with livebirth, time to biochemical pregnancy leading to live birth was defined as the number of days between enrollment and the date of hCG test. Women without livebirth were censored at 180 days (A) and 365 days(B).

\section{Supplementary Files}

This is a list of supplementary files associated with this preprint. Click to download.

- Table2and4.docx 